\title{
Lack of Evidence for Decreased Protein Stability in the 2397 (Met) Haplotype of the Leucine Rich Repeat Kinase 2 Protein Implicated in Parkinson's Disease
}

\author{
Ryan S. Anderton1,2,3*, Lauren Hill2, Rhiannon Morris ${ }^{2}$, Frank L. Mastaglia ${ }^{2,3,4}$, \\ Wayne Greene ${ }^{5}$, Sherif Boulos ${ }^{2,3}$ \\ ${ }^{1}$ School of Health Sciences and Institute for Health Research, University of Notre Dame Australia, Fremantle, WA, USA \\ ${ }^{2}$ Perron Institute for Neurological and Translational Sciences, Nedlands, WA, USA \\ ${ }^{3}$ Centre for Neuromuscular and Neurological Disorders, University of Western Australia, Nedlands, WA, USA \\ ${ }^{4}$ Institute for Immunology and Infectious Diseases, Murdoch University, Perth, WA, USA \\ ${ }^{5}$ School of Veterinary and Life Sciences, Murdoch University, Perth, WA, USA \\ Email: *ryan.anderton@nd.edu.au
}

How to cite this paper: Anderton, R.S., Hill, L., Morris, R., Mastaglia, F.L., Greene, W. and Boulos, S. (2017) Lack of Evidence for Decreased Protein Stability in the 2397 (Met) Haplotype of the Leucine Rich Repeat Kinase 2 Protein Implicated in Parkinson's Disease. Advances in Parkinson's Disease, 6, 113-123.

https://doi.org/10.4236/apd.2017.64012

Received: September 13, 2017

Accepted: November 10, 2017

Published: November 13, 2017

Copyright $\odot 2017$ by authors and Scientific Research Publishing Inc. This work is licensed under the Creative Commons Attribution International License (CC BY 4.0).

http://creativecommons.org/licenses/by/4.0/

(c) (i) Open Access

\begin{abstract}
Missense mutations in the leucine rich repeat kinase 2 (LRRK2) gene are the leading genetic cause of autosomal dominant familial Parkinson's disease. We previously reported that two mutations within the ROC domain, namely R1441C and A1442P, exhibit increased protein degradation leading to lowered steady state LRRK2 protein levels in HEK293 cells. More recently, the common WD40 domain LRRK2 haplotype, Met2397, which is a risk factor for Crohn's disease, has been shown to lower steady state protein levels in HEK293 cells. In view of recent evidence implicating LRRK2 and inflamemation in PD, we investigated the effects of Met2397 on LRRK2 expression, and compared them to the Thr2397 variant and other LRRK2 mutants. In this study, we transfected HEK293 cells with plasmid constructs encoding the different LRRK2 variants, and analyzed the resulting protein levels by Western blot and flow cytometry. Here we found that both the Met2397 and Thr2397 haplotypes yield similar levels of LRRK2 protein expression and do not appear to impact cell viability in HEK293 cells, compared to other LRRK mutants. Thus, we have concluded that the Met2397 haplotype is unlikely to play a role in LRRK2 mediated or idiopathic PD.
\end{abstract}

\section{Keywords}

Parkinson's Disease, LRRK2, Met2397, Thr2397, A1442P, R1441C 


\section{Introduction}

Parkinson's disease (PD) is a neurodegenerative condition characterised by Lewy Bodies and the loss of dopaminergic neurons within the substantian igrapars compacta [1]. While the majority of PD cases are idiopathic, causative gene mutations have been identified in about $5 \%-10 \%$ of cases [2]. Of these, missense mutations within the leucine-rich repeat kinase 2 (LRRK2) gene appear to account for the majority of familial cases [3]. The leucine-rich repeat kinase 2 protein is a member of the ROC kinase family and contains several domains including a kinase, a GTPase (ROC/Ras of Complex), COR (Carboxyl-terminus of ROC) and multiple protein-protein interaction domains [4]. Pathogenic mutations within the LRRK2 ROC domain account for around 10\% of all LRRK2 mutations, and most commonly affect codon 1441, for which three different amino acid substitutions (R1441C/G/H) have been identified [4].

We previously reported that the ROC domain missense mutation A1442P, identified in a Western Australian patient, caused reduced LRRK2 steady state protein levels, as did the common R1441C mutation [5]. Mutations within the ROC domain of $L R R K 2$ reduce GTP hydrolysis and $L R R K 2$ protein autophosphorylation, which leads to dysregulated organelle degradation, altered signalling and ultimately immune dysfunction [1] [6] [7]. However, the effects of mutations and polymorphisms in other domains of LRRK2, such as the WD40 domain, are less understood. The WD40 appears to function as a scaffold for protein-protein complexes in the formation of homodimers, for vesicle trafficking, signal transduction, cytoskeleton assembly and autophosphorylation [8]. Therefore, unsurprisingly, several mutations, or deletion, of this domain have been found to contribute to Parkinson's disease [9] [10]. Furthermore, cell death is also regulated by the WD40 domain, as neurotoxicity is reduced in mutants lacking the WD40 domain [10].

Recently, it was reported that a haplotype (Met2397) variant within the WD40 domain causes reduced protein stability and may increase susceptibility to Crohn's disease, a serious inflammatory bowel disease [11]. As the Met2397 haplotype occurs in approximately $50 \%$ of the population, we hypothesized that the Met2397 haplotype may lead to altered LRRK2 stability and may be a genetic modifier in PD. To investigate this proposition, we derived $L R R K 2$ variants of each haplotype and determined the effects on steady state protein levels in HEK293 cells using flow cytometry and Western blot analysis.

\section{Materials and Methods}

\subsection{Site-Directed Mutagenesis}

The LRRK2 Met2397 haplotype was derived by site directed mutagenesis as previously described [5], using a plasmid pCDNA3.1 encoding the GFP-LRRK2FLAG Thr2397 haplotype as the template. The primer pairs were as follows: forward 
5'-GTGCACTTTTTAAGGGAGGTAAT-GGTAAAAGAAAACAAGGAATGA-3' and reverse

5'-TGATTCCTTGTTTTCTTTTACC-ATTACCTCCTTAAAAAGTGCAC-3'. To confirm the site directed mutagenesis, the resultant constructs were fully sequenced. The other plasmid constructs used in this study are previously described [5].

\subsection{Cell Culture and Transfection of HEK293 Cells}

Human embryonic kidney 293 (HEK293) cells were cultured in DMEM (Invitrogen, Melbourne, Australia) supplemented with penicillin (20 units $/ \mathrm{ml}$ ), streptomycin $(20 \mathrm{mg} / \mathrm{ml})$, glutamine $(4 \mathrm{mM}), 5 \%$ foetal calf serum (FCS), and maintainedat $37^{\circ} \mathrm{C}\left(5 \% \mathrm{CO}_{2}\right)$. For transfection, HEK293 cells $(150,000)$ were seeded (24-well format) 24 hours prior to transfection with approximately 200 $\mu \mathrm{M}$ of LRRK2 DNA constructs. Cultures were transfected using Lipofectamine $2000^{\circledR}$ in OPTI-MEM serum-free media (Invitrogen) according to the manufacturers recommendations. DMEM was added to cells 3 hours post-transfection. Fluorescent imaging was undertaken as previously described [12]. Briefly, transfected HEK293 were fixed and visualized for GFP fluorescence (Olympus IX70; Olympus DP70 digital camera).

\subsection{RNA Extraction and RT-PCR}

Total RNA was harvested using TRIzol (Invitrogen) according to the manufacturer's instructions. One microgram of total RNA was reverse transcribed using M-MLV reverse transcriptase (Promega, Madison, WI, USA). Polymerase chain reaction was used to amplify expressed $L R R K 2$ transcripts using forward (GGTGAACCGATCGAGCTG) and reverse

(CTCCAGGATTTGGACCAGCG) primers. For internal controls, expressed GAPDH forward (AGAAGGCTGGGGCTCATTTG) and reverse

(AGGGGCCATCCACAT-CTTC) primers were used. The amplification protocol followed $55^{\circ} \mathrm{C}$ for $30 \mathrm{~min}, 95^{\circ} \mathrm{C}$ for $2 \mathrm{~min}$ and $28 \mathrm{cycles}$ of $95^{\circ} \mathrm{C}$ for $30 \mathrm{~s}$, $51^{\circ} \mathrm{C}$ for $30 \mathrm{sec}$ and $72^{\circ} \mathrm{C}$ for $45 \mathrm{sec}$.

\subsection{Flow Cytometry}

HEK293 cells were collected 72 hours post-transfection and washed with phosphate-buffered saline (PBS). Cell viability was determined by adding 7AAD (7 amino actinomycin D; $500 \mathrm{nM}$; BD Biosciences; San Jose, USA), for 15 minutes at room temperature. Cell suspensions were processed by using FACSCalibur (BD Biosciences) loaded with Cell Quest software (BD Biosciences), and GFP expressing cells were analysed with FlowJo software (version 9.1). A minimum of $1 \times 10^{5}$ cells were processed per sample and forward scatter (FSC) and side scatter (SSC) detectors were used to measure cell size and gate cell populations for analysis. The number of GFP expressing cells was calculated as the percentage of green fluorescent cells (live and dead) in the total gated cell population. 


\subsection{Western Blot Analysis}

For protein extraction, cultured HEK293 cells were lysed in buffer $(50 \mathrm{mM}$ Tris- $\mathrm{HCl}, \mathrm{pH}$ 7.5, $100 \mathrm{mM} \mathrm{NaCl}, 20 \mathrm{mM}$ EDTA, 0.1\% SDS, 0.2\% deoxycholic acid), containing complete protease inhibitor (Roche, Indianapolis, USA). Western blotting has been described previously by Anderton, Meloni [13]. Briefly, membranes were blocked in PBS-Tween $20(0.1 \%)$-containing ovalbumin (1 $\mathrm{mg} / \mathrm{ml}$ ) for 1 hour and incubated in LRRK2 (1:5000; Michael J Fox Foundation; New York, USA), GFP (1:5000; Clontech, Palo Alto, CA), FLAG (1:25,000; Sigma-Aldrich, St. Louis, USA), or CYPA (1:40,000; Biomol, Plymouth Meeting, PA) primary antibodies diluted in PBS-T $(0.1 \%)$ plus ovalbumin $(1 \mathrm{mg} / \mathrm{ml})$. Proteins were detected using a HRP-complexed secondary antibody (1:15,000 1:35,000 donkey anti-rabbit/sheep anti-mouse; GE Healthcare, Piscataway, NJ, USA), and visualized using ECL Plus (Bio-Rad, Hercules, USA). Quantification and band densitometry of Western blots was undertaken using Image J (NIH) software.

\subsection{Statistical Analysis}

Data was analysed using the Statistical Package for Social Sciences (SPSS) software, version 24 (IBM Corporation). A univariate analysis of variance (ANOVA) was conducted to investigate the main effects of LRRK2 variants, with transfection $(\mathrm{GFP}+)$ and cell viability $(\mathrm{GFP}+/ 7 \mathrm{AAD}+)$ as the dependent variables. For both ANOVAs, if main effects were observed, Bonnferoni post-hoc tests were used to investigate the simple effects (i.e. between LRRK2 variants). For all data, a $p$ value threshold of $<0.05$ was considered statistically significant.

\section{Results}

\subsection{Generation of a Met2397 LRRK2 Plasmid Construct}

A pcDNA3.1 LRRK2 expression plasmid vector (Origene; Rockville, MD, USA) fused with the N-terminus green fluorescent protein (GFP) sequence and FLAG peptide sequence has previously been described [5]. The pcDNA3.1 Thr2397 $L R R K 2$ construct was used as a template for site-directed mutagenesis to produce the Met2397 LRRK2 haplotype. The LRRK2 expression constructs used in this study (Figure 1(a)) include the threonine wild type (Thr2397), methionine wild type (Met2397), threonine R1441C (R1441C) and threonine A1442P (A1442P).

\subsection{Assessment of LRRK2 Expression in HEK293 Cells}

To determine if the Met2397 haplotype affects LRRK2 protein stability, HEK293 cells were transfected with equivalent concentrations of plasmid DNA encoding the four constructs. Fluorescence imaging of transfected HEK293 cells confirmed LRRK2 expression at 48 and 72 hours post-transfection (Figure 1(b)). The level of GFP expression in transfected cells appeared to be comparable between the LRRK2 Thr2397 and Met2397 haplotypes. In comparison and 


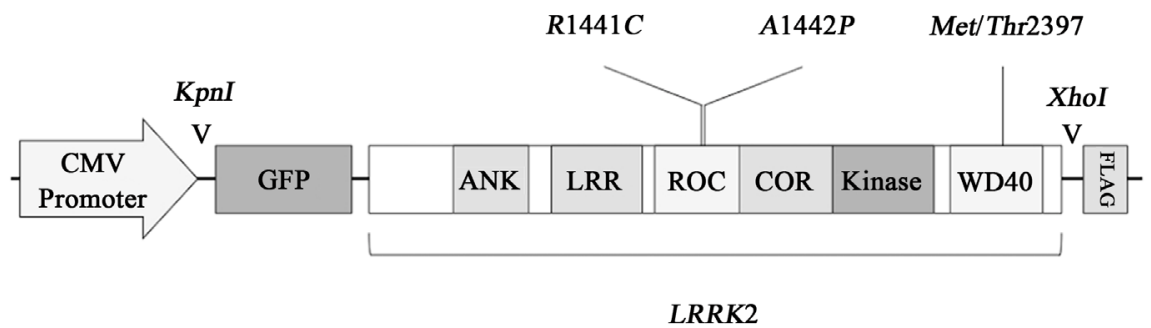

(a)

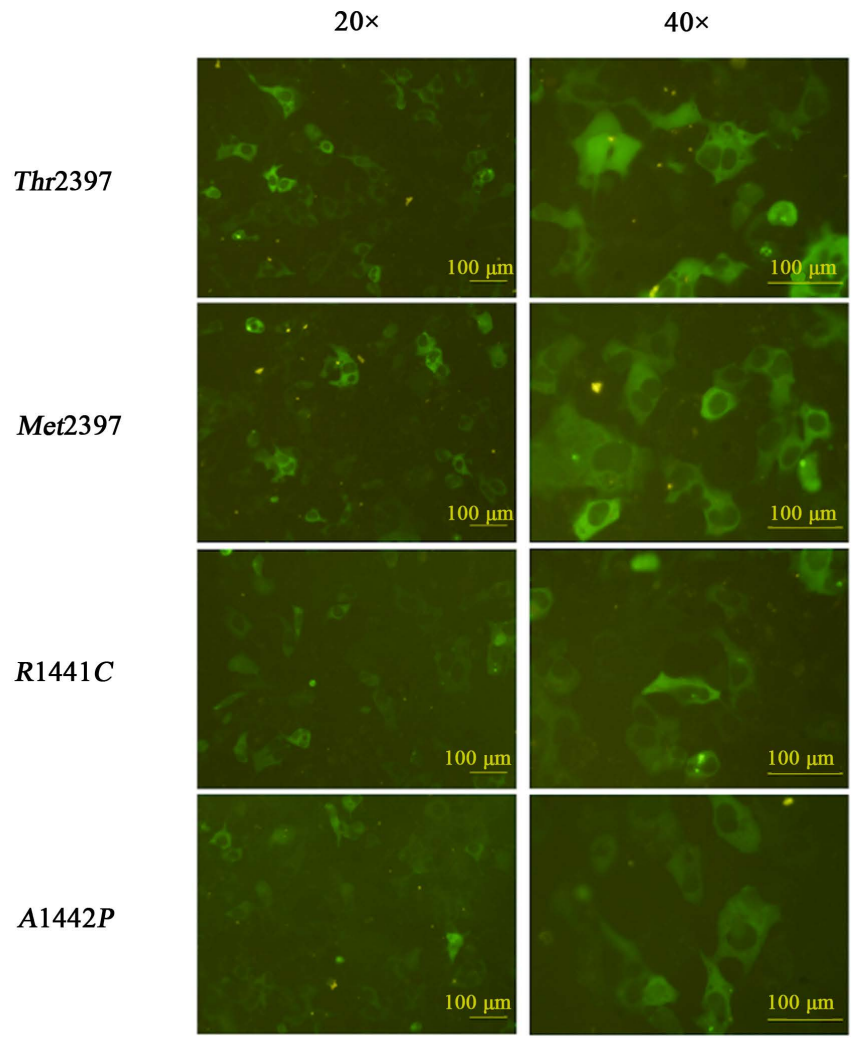

(b)

Figure 1. Confirmation of LRRK2 expression. (a) Schematic illustrating the LRRK2 plasmid constructs containing a GFP and FLAG sequences. The methionine and threonine haplotypes are shown at position 2397 in the WD40 domain. The pathogenic mutation, R1441C, and previously identified, A1442P, were also used in this study. Fluorescence microscopy of transfected HEK293 cells; (b) Fluorescence microscopy of wild type Thr2397, wild type Met2397, R1441C and A1442P LRRK2 transfected HEK293 images at 72 hours.

consistent with our previous study, GFP levels appeared to be reduced in R1441C and A1442P transfected HEK293 cells (Figure 1(b)) [5].

\subsection{Western Blot Analysis of LRRK2 Protein Levels in HEK293 Cells}

Western analysis of LRRK2 protein levels at 72 hours post-transfection showed comparable protein levels for both the Met2397 and Thr2397 wild type LRRK2 haplotypes (Figure 2(a)). Notably, following transfection with R1441C and 


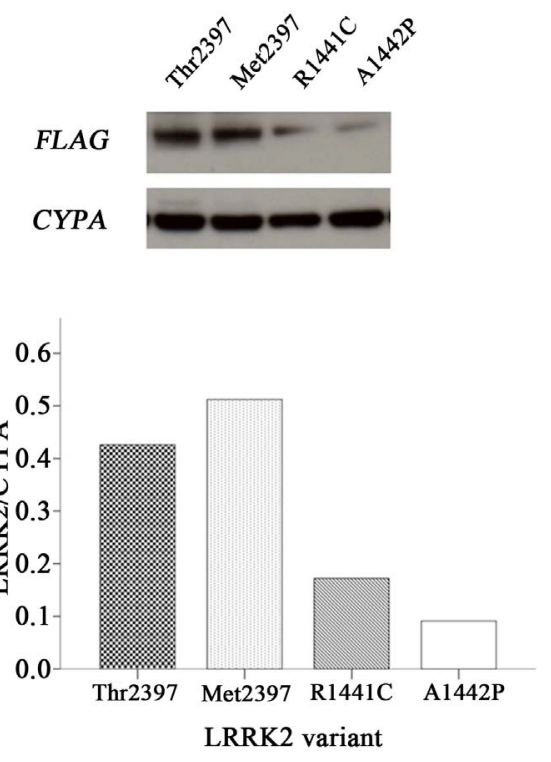

(a)

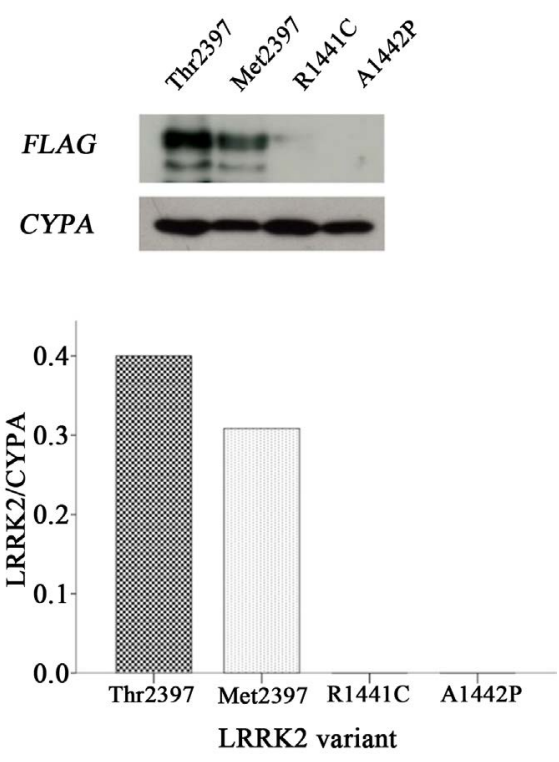

(b)

Figure 2. Western blot analysis of transfected HEK293 cells.HEK293 cells were probed for FLAG 72 hours after transfection with (a) GFP-fused and (b) non-GFP fused wild type Thr2397, wild type Met2397, R1441C and A1442P LRRK2 constructs. Data shown are representative blots and accompanying densitometry $(n=3)$.

A1442P mutants, LRRK2 protein levels were significantly less compared to both the Thr2397 and Met2397 haplotype ( $p<0.05$; Figure 2). To ensure GFP fusion was not impacting on protein stability, HEK293 cells were transfected with non-GFP fused LRRK2 construct variants. Western blot analysis of non-GFP fused LRRK2 proteins (Figure 2(b)) revealed a similar amount of expression compared to GFP-LRRK2 fused protein, confirming that that the GFP tag did not affect LRRK2 stability.

\subsection{Assessment of LRRK2 Protein Levels in HEK293 Cells Using Flow Cytometry}

To quantify LRRK2 protein expression, transfected HEK293 cells were subjected to flow cytometry at 72 hours post-transfection. To ensure equivalent concentrations of plasmid DNA were transfected into cells, we performed semi-quantitative RT-PCR (Figure 3(a)). The percentage of GFP positive cells following transfection with the LRRK2 haplotypes was 28.4\% (Thr2397) and 27.3\% (Met2397), which confirmed our Western blot data, that protein levels were equivalent. In contrast, the percentage of HEK293 cells expressing GFP was significantly reduced in cultures transfected with the mutant LRRK2 constructs R1441C (22.6\%: $p<0.05)$ and A1442P (16.9\%: $p<0.001)$. The geometric mean of fluorescence (GMF) for GFP did not significantly differ between HEK293 cells transfected with the Met2397 and Thr2397 LRRK2 haplotypes (Table 1). However, there was a significant difference between the wild type haplotypes and R1441C $(p<0.05)$ and A1442P $(p<0.001)$. 


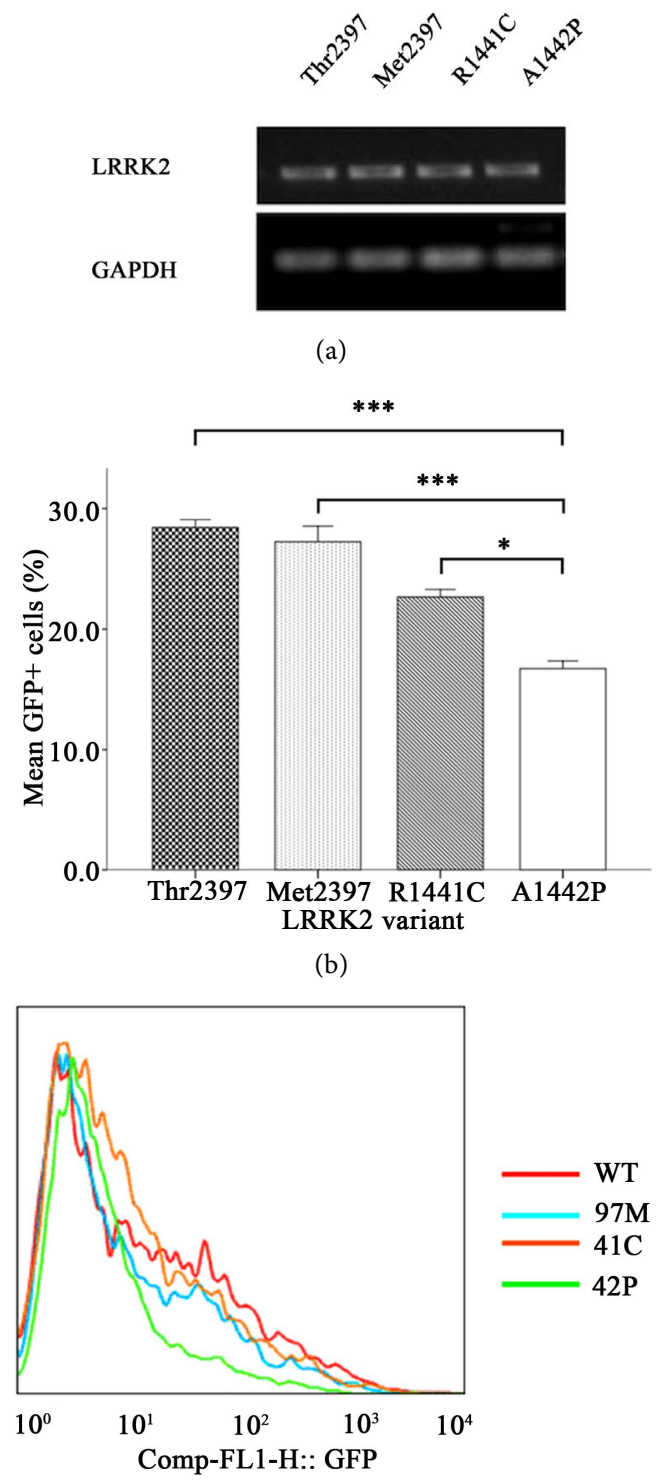

(c)

Figure 3. Flow cytometry analysis of transfected HEK293 cells. (a) Semi-quantitative RT-PCR analysis of transfected LRRK2 pDNA with GAPDH control mRNA levels; (b) HEK cells transfected with wild type GFP-fused Thr2397, wild type Met2397, R1441C and A1442P LRRK2 constructs; (c) A representative histogram showing relative LRRK2 expression as a function of GFP fluorescence for all the expression constructs. Data shown are the mean \pm standard error; ${ }^{\star} p<0.05,{ }^{* * *} p<0.001(n=4)$.

Table 1. Average geometric mean fluorescence (GMF) values following LRRK2 construct transfection.

\begin{tabular}{cc}
\hline LRRK2 construct & GMF (SD \pm ) \\
\hline Thr2397 & $27.94(2.98)$ \\
Met2397 & $28.53(3.94)$ \\
R1441C & $24.25(1.62)$ \\
A1442P & $19.59(2.65)$
\end{tabular}




\subsection{The Effect of the LRRK2 2397 Haplotype on HEK293 Cell Viability}

To determine if type LRRK2 2397 haplotype impacts on cell viability, transfected HEK293 cells were stained with 7AAD to determine non-viable cell populations. Expression of the wild type LRRK22397 haplotypes resulted in comparable rates of non-viable cells (Figure 4). In contrast, expression of both ROC domain mutants increased cell death. The percentage of cell death in HEK293 cultures transfected with the A1442P and R1441C constructs were significantly higher compared to both wild type haplotypes $(p<0.005)$.

\section{Discussion}

Previous studies have suggested that changes to the LRRK2 amino acid sequence can significantly affect protein function and stability, potentially contributing to PD pathogenesis [5] [14]. Recently, our laboratory reported that mutations in the LRRK2 ROC domain led to a decrease in protein stability, causing a proteasome-mediated degradation to occur more rapidly [5]. The WD40 domain of LRRK2 appears to be important in protein-protein interaction, cytoskeletal activity, and promoting neuronal survival [8]. The common haplotype within the WD40 domain, Met2397, has been linked to immune dysfunction in Crohn's disease [15] due to reduced protein stability, as is also seen with other LRRK2 domain mutations [11].

This study investigated the stability of the LRRK2 Met2397 haplotype along with two previously described ROC domain missense mutations (R1441C and A1442P). Fluorescent microscopy transfected HEK293 cells revealed that levels of LRRK2 protein did not vary between wild type Met2397 and Thr2397

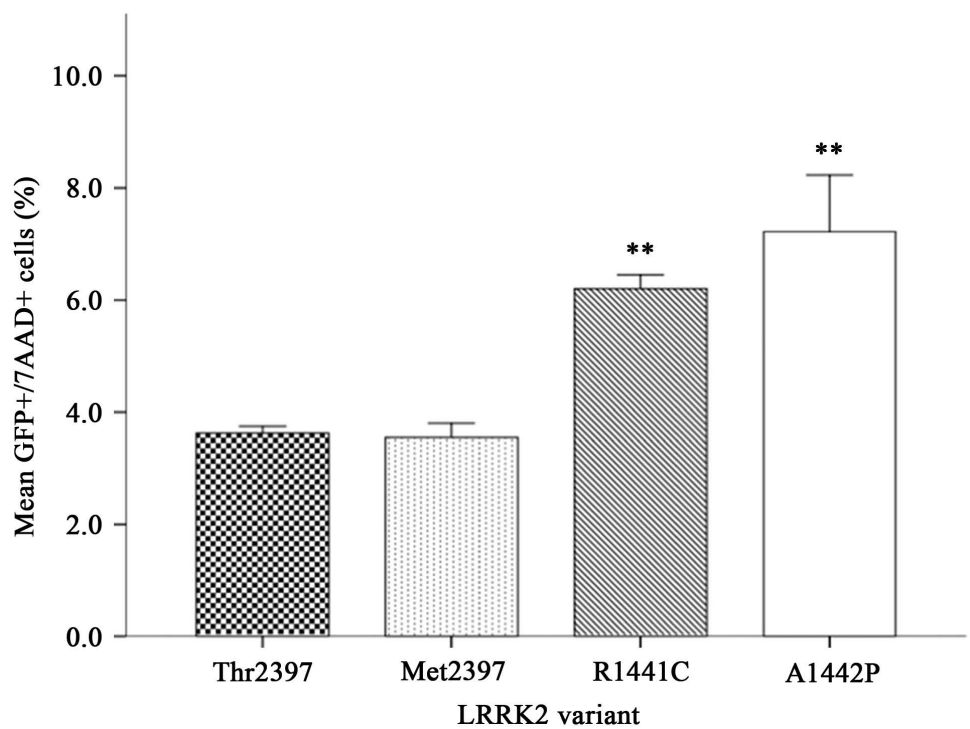

Figure 4. Determination of non-viable cells in HEK293 cultures transfected with LRRK2 constructs. Non-viable cells were determined by positive staining with 7AAD. Transfected non-viable cells $(\mathrm{GFP}+/ 7 \mathrm{AAD}+)$ were analysed by flow cytometry, with results shown as levels of cell death. Data shown are the mean \pm standard error; ${ }^{* *} p<0.005(n=3)$. 
haplotypes. Furthermore, Western blot analysis of FLAG demonstrated comparable protein levels when comparing wild type haplotypes. Surprisingly, these results contradict previous findings that showed expression of the wild type LRRK2 Met2397 haplotype produced a less stable protein than the Thr2397 haplotype, when expressed in HEK293 cells [11]. Further, LRRK2 protein levels were reduced in the peripheral blood B-cells of patients homozygous for the Met2397 haplotype [11]. In order to further investigate the disparity between our findings and those of Liu et al. (2011), LRRK2 protein levels were quantified using flow cytometry. Similarly, there were no differences in mean geometric fluorescence or GFP positive cells 72 hours after transfection with Met2397 or Thr2397 LRRK2 constructs. One possible cause for such findings in the present study is the difference in expression constructs. However, use of a previously validated and sequenced construct [5] supports the current findings, suggesting a presently unknown mechanism for the contradictory results.

This study also investigated the effect of ROC and WD40 mutations on LRRK2 protein stability, and subsequent cell viability. As previously stated, the WD40 domain functions in vesicle trafficking, signal transduction, cytoskeleton assembly and autophosphorylation [8]. Most importantly, the WD40 domain has been linked to cell viability, as neurotoxicity is reduced in mutants lacking the WD40 domain [10]. The missense mutation, G2385R, which is located in the WD40 domain close to the 2397 codon, reportedly reduces the cell's ability to handle oxidative stress, impacting on cell survival [16]. Following flow cytometry analysis, 7AAD staining of LRRK2 expressing HEK293 cells was used to quantify levels of cell death. Our results showed no differences between the wild type Met2397/Thr2397 haplotypes, suggesting the Met2397 haplotype is not directly implicated in the neuronal loss seen in Parkinson's disease.

Previous studies into the LRRK2 protein have identified the ROC domain as being important in the maintenance of protein stability [17]. In this study, HEK293 cells expressing R1441C and A1442P ROC mutants showed reduced viability, in comparison to wild type Met2397/Thr2397 haplotypes. A decrease in cell viability has been linked to the R1441C mutation and other LRRK2 missense mutations including the Y1699C, G2019S and I2020T mutations, in both immortalised and primary cells [7] [14] [18] [19].

Interestingly, the LRRK2 protein has also been linked to the regulation of immune signalling, such as those mediated by the Toll-like Receptor (TLR) in microglia and B cells, which cause the release of TNF $\alpha$ and interleukin- 6 in response to pathogens [20]. The LRRK2 protein has been reported to down-regulate the NFAT signalling pathway, and LRRK2 knockout in vivo results in the over-production of inflammatory cytokines [11]. Indeed, the R1441G ROC domain missense mutation is also reported to increase inflammatory cytokine release [21]. Therefore, the reduction in cellular LRRK2 protein levels could be directly linked to the over-production of inflammatory cytokines detected in the brains and spinal fluid of PD patients [22] [23] [24]. 
In this study, we have confirmed that the ROC domain missense mutations, R1441C and A1442P, decrease LRRK2 protein stability. Furthermore, for the first time this study demonstrates that the A1442P missense mutation causes decreased cell viability in HEK293 cells. However, the results from this study indicate that the Met2397 haplotype does not impact LRRK2 protein stability or cell viability, conflicting with previous reports by Liu et al. (2011). While the present study was limited by ectopic expression of LRRK2, both GFP and non-GFP fused LRRK2 constructs revealed similar findings on protein stability. In conclusion, we have investigated the impact of the Met2397/Thr2397 haplotype and provided further evidence for the involvement of the ROC domain in LRRK2 protein survival. These findings suggest the LRRK2 Met2397 haplotype variation is unlikely to play a significant role in the pathogenesis of $L R R K 2$-mediated PD.

\section{Acknowledgements}

The authors declare no conflict of interest and acknowledge the Centre for Microscopy, Characterisation and Analysis (University of Western Australia) for technical assistance. This work was supported by the University of Notre Dame Australia and the Rebecca Cooper Foundation.

\section{References}

[1] Schapira, A.H. and Jenner, P. (2011) Etiology and Pathogenesis of Parkinson's Disease. Movement Disorders, 26, 1049-1055. https://doi.org/10.1002/mds.23732

[2] Corti, O., Lesage, S. and Brice, A. (2011) What Genetics Tells Us about the Causes and Mechanisms of Parkinson's Disease. Physiological Reviews, 91, 1161-1218.

[3] Smith, W.W., et al. (2005) Leucine-Rich Repeat Kinase 2 (LRRK2) Interacts with Parkin, and Mutant LRRK2 Induces Neuronal Degeneration. Proceedings of the National Academy of Sciences of the United States of America, 102, 18676-18681. https://doi.org/10.1073/pnas.0508052102

[4] Mills, R.D., et al. (2014) Prediction of the Repeat Domain Structures and Impact of Parkinsonism-Associated Variations on Structure and Function of all Functional Domains of Leucine-Rich Repeat Kinase 2 (LRRK2). Human Mutation, 35, 395-412. https://doi.org/10.1002/humu.22515

[5] Greene, I.D., et al. (2014) Evidence that the LRRK2 ROC Domain Parkinson's Disease-Associated Mutants A1442P and R1441C Exhibit Increased Intracellular Degradation. Journal of Neuroscience Research, 92, 506-516.

https://doi.org/10.1002/jnr.23331

[6] West, A.B., et al. (2005) Parkinson's Disease-Associated Mutations in Leucine-Rich Repeat Kinase 2 Augment Kinase Activity. Proceedings of the National Academy of Sciences of the United States of America, 102, 16842-16847. https://doi.org/10.1073/pnas.0507360102

[7] Greggio, E., et al. (2006) Kinase Activity Is Required for the Toxic Effects of Mutant LRRK2/Dardarin. Neurobiology of Disease, 23, 329-341. https://doi.org/10.1016/j.nbd.2006.04.001

[8] Gilsbach, B.K. and Kortholt, A. (2014) Structural Biology of the LRRK2 GTPase and Kinase Domains: Implications for Regulation. Frontiers in Molecular Neuroscience, 7. 
[9] Sheng, D., et al. (2010) Deletion of the WD40 Domain of LRRK2 in Zebrafish Causes Parkinsonism-Like Loss of Neurons and Locomotive Defect. PLoS Genet, 6 , e1000914.

[10] Jorgensen, N.D., et al. (2009) The WD40 Domain Is Required for LRRK2 Neurotoxicity. PLoS One, 4, e8463.

[11] Liu, Z., et al. (2011) The Kinase LRRK2 Is a Regulator of the Transcription Factor NFAT That Modulates the Severity of Inflammatory Bowel Disease. Nat Immunol, 12, 1063-1070. https://doi.org/10.1038/ni.2113

[12] Anderton, R.S., et al. (2014) Investigation of a Recombinant SMN Protein Delivery System to Treat Spinal Muscular Atrophy. Translational Neuroscience, 5, 8-16. https://doi.org/10.2478/s13380-014-0201-2

[13] Anderton, R.S., et al. (2011) Survival of Motor Neuron Protein Over-Expression Prevents Calpain-Mediated Cleavage and Activation of Procaspase-3 in Differentiated Human SH-SY5Y Cells. Neuroscience, 181, 226-233. https://doi.org/10.1016/j.neuroscience.2011.02.032

[14] Ohta, E., Kubo, M. and Obata, F. (2010) Prevention of Intracellular Degradation of I2020T Mutant LRRK2 Restores Its Protectivity against Apoptosis. Biochemical and Biophysical Research Communications, 391, 242-247. https://doi.org/10.1016/j.bbrc.2009.11.043

[15] Barrett, J.C., et al. (2008) Genome-Wide Association Defines More than 30 Distinct Susceptibility Loci for Crohn's Disease. Nature Genetics, 40, 955-962. https://doi.org/10.1038/ng.175

[16] Tan, E.K., et al. (2007) The LRRK2 Gly2385Arg Variant Is Associated with Parkinson's Disease: Genetic and Functional Evidence. Hum Genet, 120, 857-863. https://doi.org/10.1007/s00439-006-0268-0

[17] Carlessi, R., et al. (2011) GTP Binding to the ROC Domain of DAP-Kinase Regulates Its Function through Intramolecular Signalling. EMBO Reports, 12, 917-923. https://doi.org/10.1038/embor.2011.126

[18] Smith, W.W., et al. (2006) Kinase Activity of Mutant LRRK2 Mediates Neuronal Toxicity. Nature Neuroscience, 9, 1231-1233. https://doi.org/10.1038/nn1776

[19] MacLeod, D., et al. (2006) The Familial Parkinsonism Gene LRRK2 Regulates Neurite Process Morphology. Neuron, 52, 587-593.

https://doi.org/10.1016/j.neuron.2006.10.008

[20] Dzamko, N. and Halliday, G.M. (2012) An Emerging Role for LRRK2 in the Immune System. Biochemical Society Transactions, 40, 1134-1139. https://doi.org/10.1042/BST20120119

[21] Gillardon, F., Schmid, R. and Draheim, H. (2012) Parkinson's Disease-Linked Leucine-Rich Repeat Kinase 2(R1441G) Mutation Increases Proinflammatory Cytokine Release from Activated Primary Microglial Cells and Resultant Neurotoxicity. Neuroscience, 208, 41-48. https://doi.org/10.1016/j.neuroscience.2012.02.001

[22] Nagatsu, T., et al. (2000) Changes in Cytokines and Neurotrophins in Parkinson's Disease. Journal of Neural Transmission. Supplementa, 60, 277-290.

[23] Sawada, M., Imamura, K. and Nagatsu, T. (2006) Role of Cytokines in Inflammatory Process in Parkinson's Disease. Journal of Neural Transmission. Supplementa, 70, 373-381.

[24] Long-Smith, C.M., Sullivan, A.M. and Nolan, Y.M. (2009) The Influence of Microglia on the Pathogenesis of Parkinson's Disease. Progress in Neurobiology, 89, 277-287. https://doi.org/10.1016/j.pneurobio.2009.08.001 